\title{
Dodging death
}

The expression of apoptosisstimulating protein of $\mathrm{p} 532$ (ASPP2, also known as TP53BP2) is often reduced in many human cancers, and a growing body of evidence suggests that it functions as a tumour suppressor. The creation of a heterozygous Aspp2 mouse model indicates that ASPP2 is a haploinsufficient tumour suppressor and provides new insights into the mechanisms of ASPP2 function in tumorigenesis.

Kerstin Kampa and colleagues targeted mouse Aspp2 using homologous recombination, but found that Aspp $2^{-/-}$mice were embryonic lethal. They therefore focused on analysing $A s p p 2^{+/-}$mice and although Aspp $2^{+/}$ mice were normal and fertile, they showed a higher incidence of spontaneous tumours than did wild-type mice. When the authors investigated ASPP2 expression in individual tumours from the Aspp2 $2^{+/-}$mice, they found no evidence for loss of heterozygosity. To investigate whether ASPP2 cooperates with p53 to inhibit tumorigenesis, Kampa et al. crossed the Aspp $2^{+/-}$mice with $\operatorname{Tr} p 53^{+/-}$mice. There was no difference in the incidence of tumour formation in Aspp $2^{+/+} \operatorname{Tr} p 53^{+/-}$and $A s p p 2^{+/-} \operatorname{Tr} p 53^{+/-}$mice, suggesting that the tumour-suppressive function of ASPP2 might involve p53-independent mechanisms. Because the expression of ASPP2 is known to be induced by cellular stress and damage, the authors subjected $A s p p 2^{+/-}$and Aspp $2^{+/+}$mice to $\gamma$-irradiation. Aspp $2^{+/+}$mice did not develop any tumours after irradiation; however, Aspp $2^{+/-}$mice developed aggressive high-grade lymphomas of thymic origin. To gain further insights into the underlying mechanisms that are responsible for the development of these lymphomas, the authors cultured primary thymocytes from the mice and exposed them to $\gamma$-irradiation. They found that $A s p p 2^{+/-}$thymocytes showed lower levels of apoptosis after $\gamma$-irradiation than $A s p p 2^{+/+}$ cells, suggesting that a block in apoptosis in the Aspp2 $2^{+/-}$cells could allow thymocytes with tumorigenic mutations to persist.

To investigate whether ASPP2 expression affects stress response pathways other than apoptosis, the authors analysed primary mouse embryonic fibroblasts (MEFs) from the $A s p p 2^{+/-}$mice after $\gamma$-irradiation. Normally, primary MEFs arrest at the G0/G1 cell cycle checkpoint after this treatment, but the Aspp2 $2^{+-} \mathrm{MEF}$

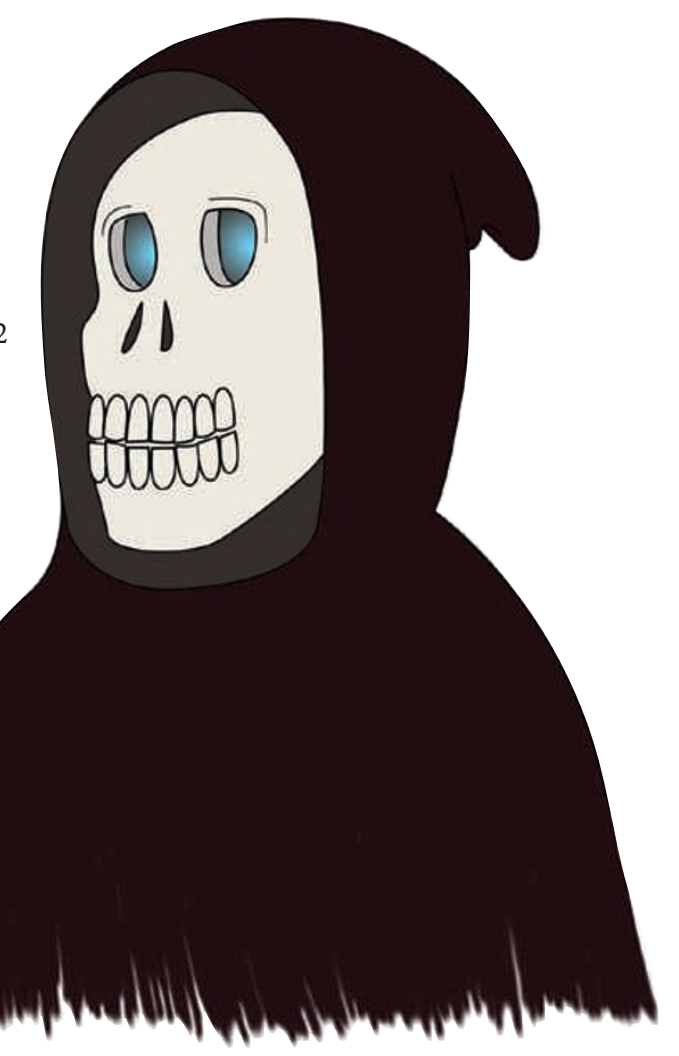

population did not show a significant increase in the number of cells in G0/G1. This result indicates that ASPP2 function is more complex than just enhancing pro-apoptotic programmes, and expands the list of potential mechanisms by which ASPP2 acts as a tumour suppressor.

Meera Swami

ORIGINAL RESEARCH PAPER Kampa, K. M. et al. Apoptosis-stimulating protein of $\mathrm{p} 53$ (ASPP2) heterozygous mice are tumor-prone and have attenuated cellular damage-response thresholds. Proc. Natl Acad. Sci. USA 26 Feb 2009 (doi:10.1073/pnas.0809080106) 